\title{
Reconnecting Rural Native Hawaiian Families to Food through Aquaponics
}

\author{
Jazmine Kaleihua Beebe ${ }^{1}$, Yvette Amshoff ${ }^{1}$, Ilima Ho-Lastimosa ${ }^{2,3,4}$, Ghazaleh Moayedi ${ }^{1}{ }^{(0)}$, \\ Asha L.C. Bradley ${ }^{1}$, Inji N. Kim ${ }^{1}$, Napua Casson ${ }^{1}$, Robert Protzman ${ }^{1}$, Danielle Espiritu ${ }^{5}$, \\ Michael S. Spencer ${ }^{6}$ and Jane J. Chung-Do ${ }^{1,2,3, *}$ \\ 1 Office of Public Health Studies, University of Hawai'i at Mānoa, Honolulu, HI 96822, USA; \\ cockettj@hawaii.edu (J.K.B.); yamshoff@hawaii.edu (Y.A.); gmoayedi@hawaii.edu (G.M.); \\ ashabradley808@gmail.com (A.L.C.B.); ikim2@hawaii.edu (I.N.K.); casson@hawaii.edu (N.C.); \\ protzman@hawaii.edu (R.P.) \\ 2 God's Country Waimānalo, Waimānalo, HI 96795, USA; ilima888@gmail.com \\ 3 Ke Kula Nui O Waimānalo, Waimānalo, HI 96795, USA \\ 4 College of Tropical Agriculture \& Human Resources, University of Hawai'i at Mānoa, Waimānalo Learning \\ Center, Waimānalo, HI 96795, USA \\ 5 College of Education, University of Hawai'i at Mānoa, Honolulu, HI 96822, USA; despirit@hawaii.edu \\ 6 School of Social Work, University of Washington, Indigenous Wellness Research Institute, Seattle, WA 98105, \\ USA; mspenc@uw.edu \\ * Correspondence: chungjae@hawaii.edu
}

Received: 30 November 2019; Accepted: 9 January 2020; Published: 15 January 2020

check for updates

\begin{abstract}
Food insecurity is a pressing issue in Hawai' $i$ as the vast majority of available and accessible foods are imported. To address this issue, a backyard aquaponics program was implemented from 2010 to 2016 to offer additional avenues to food sovereignty in a rural predominantly Native Hawaiian community. Aquaponics provides a contained and sustainable food production system that models Native Hawaiian principles of land and water stewardship. The purpose of this community-engaged study was to identify the outcomes and resources needed to continue sustaining the backyard aquaponics systems. The researchers began building a relationship with the community by helping to build several aquaponics systems. The researchers and community partner co-developed the interview questions and participants were interviewed in-person. The outcomes of the study revealed multiple benefits of having a backyard aquaponics system, including increased access to vegetables and fruit, improved diet, low maintenance cost, and enhanced family and community connectedness. Participants reported a renewed connection to Native Hawaiian values, especially land stewardship. Challenges included leaks and breakages with the system, overproduction of fish, complications in water temperature, and vulnerability to unpredictable weather. These findings suggest that backyard aquaponics systems have the potential to provide multiple benefits including alleviating barriers related to food security.
\end{abstract}

Keywords: community-based participatory research; food security; nutrition; community garden; Hawaii; indigenous; qualitative

\section{Introduction}

Surrounded by vast bodies of water and approximately 2500 miles from the nearest land mass, Kanaka Maoli or Native Hawaiians, the indigenous peoples of Hawai'i, developed a deep and knowledgeable relationship with the environment. Survival in the middle of the Pacific Ocean required understanding, managing, and maintaining Hawai'i's natural resources. This consciousness was fundamental to the traditions and practices of Native Hawaiians and expressed through the core values 
of pono (righteousness) and lōkahi (balance and harmony) (Rezentes 1996). It is through these values that Native Hawaiians secured and stabilized their finite resources to perpetuate a healthy people and environment. However, western colonization and the illegal overthrow of the Hawaiian Kingdom in 1893 dramatically changed the people, culture, and lifestyles of Hawai'i (Kame'eleihiwa 1992). Land in Hawai' $i$ was privatized through what is known as the Māhele of 1848. While contemporary scholars acknowledge the ways the Māhele was an example of "Ōiwi agency" at a time of great change and uncertainty for Hawai'i, land privatization was a foreign concept at odds with Native Hawaiian traditional understanding of relationship to land (Beamer 2014; Kame'eleihiwa 1992). Therefore, many Native Hawaiians did not stake claim to ancestral lands, which created the conditions that gradually dispossessed Native Hawaiians from the land and traditional food sources. This disconnection with the land has contributed to the current health disparities faced by Native Hawaiians in the present day (Kaholokula et al. 2009).

Today, similar to other indigenous communities across the globe, Native Hawaiians disproportionately suffer from pervasive health outcomes in their own homeland. In Hawai'i, they are $130 \%$ more likely to die from diabetes and $68 \%$ more likely to die from heart disease compared to the state averages (Look et al. 2014). In addition, areas with large numbers of Native Hawaiian residents tend to lack healthy food grocers and restaurants yet have high concentrations of fast food outlets (Johnson et al. 2004; Mau et al. 2008). As degradation of the environment continues, the access to and the availability of healthy and nutritious food has been limited. This has been compounded by land dispossession that has decreased traditional subsistence practices. To address these challenges, there is a growing sense of need to return to the values of pono and lōkahi to promote environmental health, natural resource management, and food security (Ho-Lastimosa et al. 2014). In the last few decades, tremendous efforts among Native Hawaiians to revitalize traditional cultural practices are occurring. These efforts include food production, such as kalo farming and restoration of ancient loko i'a (fish ponds), to exercise their kuleana of mālama 'āina (Goodyear-Ka'ōpua et al. 2014).

According to the (World Food Programme 2016), the food aid arm of the United Nations, the definition of food security is having the availability and adequate access at all times to sufficient, safe, and nutritious food to maintain a healthy and active lifestyle. In Hawai' $i$, the concern for food security became apparent with the release of "Increased Food Security and Food Self-Sufficiency Strategy," which reported that $85-90 \%$ of Hawai' $i$ 's food is currently being imported (Department of Business Economic Development and Tourism 2012). For many Native Hawaiian communities, the approach to address food (in)security must include efforts grounded in Hawaiian customary practices, which are deeply embedded in the 'aina (land) (McGregor et al. 2003).

One such effort includes the use of backyard family aquaponics, which models the stewardship and reciprocity found in the ahupua'a system, a political-geographical land division that encompasses the Native Hawaiian food system. Each of the major Hawaiian Islands are divided into moku, large land districts. O'ahu, the most populated Hawaiian island, consists of six moku. Moku are further divided into ahupua'a, which typically extend from the uplands all the way down onto the reefs and into the sea (Kame'eleihiwa 2016). The health and aquaculture production of an ahupua'a's reefs and coastal fisheries, including loko i'a (fishponds), are directly tied to the health of its lo'i kalo (flooded taro agroecosystems) and upland forestry areas (Möhlenkamp et al. 2018). As a result, there was an understanding that kānaka (people) have the kuleana (responsibility) to maintain the health of each part of the ahupua'a, ensuring the pono and lōkahi of the entire system. Aquaponics utilizes the symbiotic relationship between fish and plants by effectively combining hydroponics (raising plants in water) and aquaculture (raising fish in tanks) to create a contained, sustainable, food production system similar to the ahupua'a system (Tokunaga et al. 2013); see Figure 1. By maintaining aquaponics systems, one is able to experience the pilina (reciprocal relationships) between land and sea as well as humans and food. 


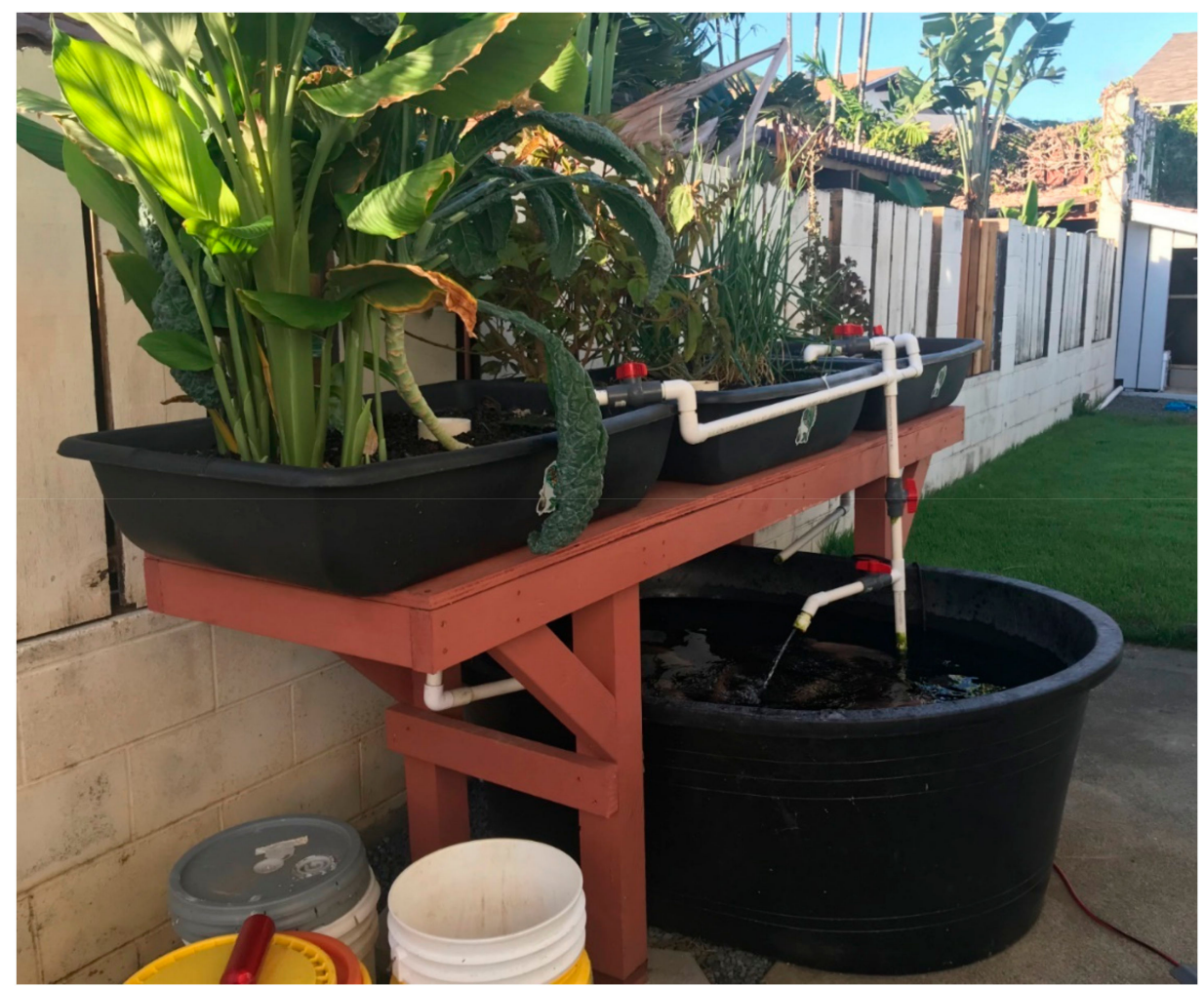

Figure 1. Backyard aquaponics system.

Aquaponics has been flourishing in the community of Waimānalo, a rural town that is located in windward O'ahu. Nearly one-third of Waimānalo residents are Native Hawaiian and the average family income is $31 \%$ less than that of the state average (U.S. Census Bureau 2015). The homeownership rate in Waimānalo (62.6\%) is higher than the state average (57.6\%), according to the (U.S. Census Bureau 2015), with over 600 Native Hawaiian families living on the Waimānalo Hawaiian Homestead. Hawaiian Homesteads were created by The Hawaiian Homes Commission Act 1920 introduced into the United States Congress by Prince Jonah Kūhiō Kalaniana'ole (Waimānalo Hawaiian Homestead Association 2019). To live on Hawaiian Homestead, residents must demonstrate that their lineage is at least $50 \%$ Hawaiian.

In Waimānalo, as well as other communities with high numbers of Native Hawaiian residents, more than $30 \%$ of households are food insecure due to the high cost of living and other socioeconomic barriers (Hawaii Department of Health 2001). Despite these challenges, it is important to recognize the many strengths and assets that reside in Waimānalo, including a number of flourishing grassroots efforts to improve the health and wellbeing of the Native Hawaiian residents. One such example is God's Country Waimānalo (GCW), a Native Hawaiian grassroots organization that was founded by Ilima Ho-Lastimosa. Rooted in cultural preservation, community partnerships, and connecting the Hawaiian culture to other aspects of health and wellness, GCW is guided by four key Native Hawaiian principles: kuleana (responsibility), mālama (to care for, to protect), 'ike pono (intellect), and ha'aha'a (humility, humbleness) (Ho-Lastimosa et al. 2014). By living out these principles, participants learn to contribute to the collective state of pono and lōkahi as a community and help to shape GCW's culturally-grounded programs.

From 2010 to 2016, GCW's founder and Executive Director, Ilima Ho-Lastimosa, obtained funding from multiple local agencies to implement a backyard aquaponics program in Waimānalo. Over this six-year period, whenever funding was obtained, a cohort of 8-10 families were invited to participate in a series of aquaponics workshops. These workshops focused on technical and cultural aspects of aquaponics, including assessing water quality, 'ai pono (healthy eating) through cooking demonstrations, and lā'au lapa'au (traditional herbal medicine). As the community heard about the 
program through word of mouth, more families expressed interest and were placed on a waiting list until another set of funding was obtained. While Waimānalo families of all ethnicities were welcomed, Native Hawaiian families were prioritized. Because all workshops used a hands-on, family-based, and collective learning approach, multi-generational members from each family were encouraged to attend the workshops together. Each cohort culminated with a "Build Weekend," where families received all of the supplies needed to build their aquaponics system. Aligned with the value of lōkahi, the families in the cohort visited each other's homes to help one another build their systems. Throughout the six years, over 70 Waimānalo families participated in the program and have built aquaponics systems in their backyards.

Recognizing the need to better understand the experiences of families with aquaponics systems, GCW collaborated with a faculty member and a group of graduate students from the University of Hawai'i at Mānoa, Office of Public Health Studies to conduct a small-scale qualitative study on the aquaponics efforts in Waimānalo. Community-based participatory research (CBPR) principles were used to guide the student researchers throughout the community engagement and research process (Israel et al. 1998). The purpose of this community-engaged study was to identify the outcomes and resources needed to continue sustaining the backyard aquaponics systems.

\section{Methods}

At the request of GCW, the University of Hawai'i student researchers conducted a qualitative study to interview Waimānalo families who participated in GCW's aquaponics program. Families who had functioning aquaponics systems, those with non-functioning systems, and those who were interested in obtaining aquaponics systems were interviewed from October to November 2016. This study was approved by the University of Hawai'i Committee of Research Compliance (CHS 2017-00092).

\subsection{Community Engagement}

In order to gain a deeper understanding of the Waimānalo community and learn about aquaponics technology, the student researchers attended a GCW aquaponics workshop. Researchers were introduced to the mechanics of a working system and maintenance of an aquaponics garden. Ilima Ho-Lastimosa also taught the student researchers how to harvest, prepare, and cook the fish from the aquaponics system. Attending this workshop informed the development of the interview guide and prepared researchers for conducting interviews in the community.

\subsection{Participants}

To capture a range of perspectives and experiences with aquaponics systems among families connected to or living in Waimānalo, recruitment focused on three groups: (1) Families currently maintaining a functioning aquaponics system; (2) Families with partially functioning or non-functioning aquaponics systems; (3) Families without an aquaponics system but interested in starting a system. GCW provided an initial contact list of potential participants who fell within the three target groups. Potential participants were contacted by phone or email to be recruited into the study and snowball sampling was used to identify further potential participants. A total of 21 families were identified and a total of nine families were interviewed.

\subsection{Measures}

A semi-structured interview guide was developed with the guidance of GCW. The questions for those who were maintaining a functioning system as well as those with a partially functioning or non-functioning system were similar. The guide included questions on actual and anticipated benefits, challenges, and needs for sustaining aquaponics systems. For example, participants were asked "Has your family's diet changed because of your aquaponics system?" and "How do you think aquaponics impacted the Waimānalo community?" to identify benefits of the intervention. Challenges of maintaining a system were identified by asking questions, such as, "What were the challenges 
you had in maintaining your system?" and "How labor intensive is it to maintain your aquaponics system?". Participants were asked about what they would need to help sustain their systems with questions, such as, "What are some resources that would help you maintain your system now?" and "What would you like to learn more about to help you be successful with the system?".

The interview questions for the third group of participants, those who were interested in an aquaponics system, focused on their personal interests in and their expectations for an aquaponics system ("How did you first hear about aquaponics?" "What kind of benefits do you hope it will bring?"), and expectations for family and community changes ("Have you thought about each family member's role in maintaining the system?" "What positive impacts do you see aquaponics bringing to the community as a whole?"). Questions were reviewed by the GCW community partner for clarity, ease of understanding, and cultural relevance. A demographic survey was also developed to capture household size, ethnicity, and the functions of their aquaponics system.

\subsection{Procedures}

Each interview was conducted with two researchers present; one researcher asked questions while the other transcribed the interview. Interviews took place at participants' homes and were 45 to $60 \mathrm{~min}$ in length. Interviews were audiotaped for transcription verification. Homemade cards were provided as cultural gifts to show appreciation for participants' time and knowledge. After conducting the interviews, the two researchers debriefed on transcription notes. Notes were organized based on the three participant groups. After each researcher coded the data individually, researchers compared codes and identified emerging themes by consensus. Once themes were identified, the researchers hosted a gathering and reported their findings back to the community partner, the participating families, and the wider community to validate themes.

\section{Results}

A total of 12 participants from nine families were interviewed in October and November 2016. Six of the families lived on the Waimānalo Hawaiian Homestead. Four families had current systems, two had non-functioning or partially-functioning systems, and three were interested in obtaining a system. A majority of participants self-identified as Native Hawaiian $(n=9)$, and others self-identified as African-American $(n=2)$ and Caucasian/White $(n=1)$. There was a total of seven females and five males. Three couples were interviewed together. A total of 12 themes emerged, which were sorted into three major categories of benefits, challenges, and participant suggestions (see Figure 2).
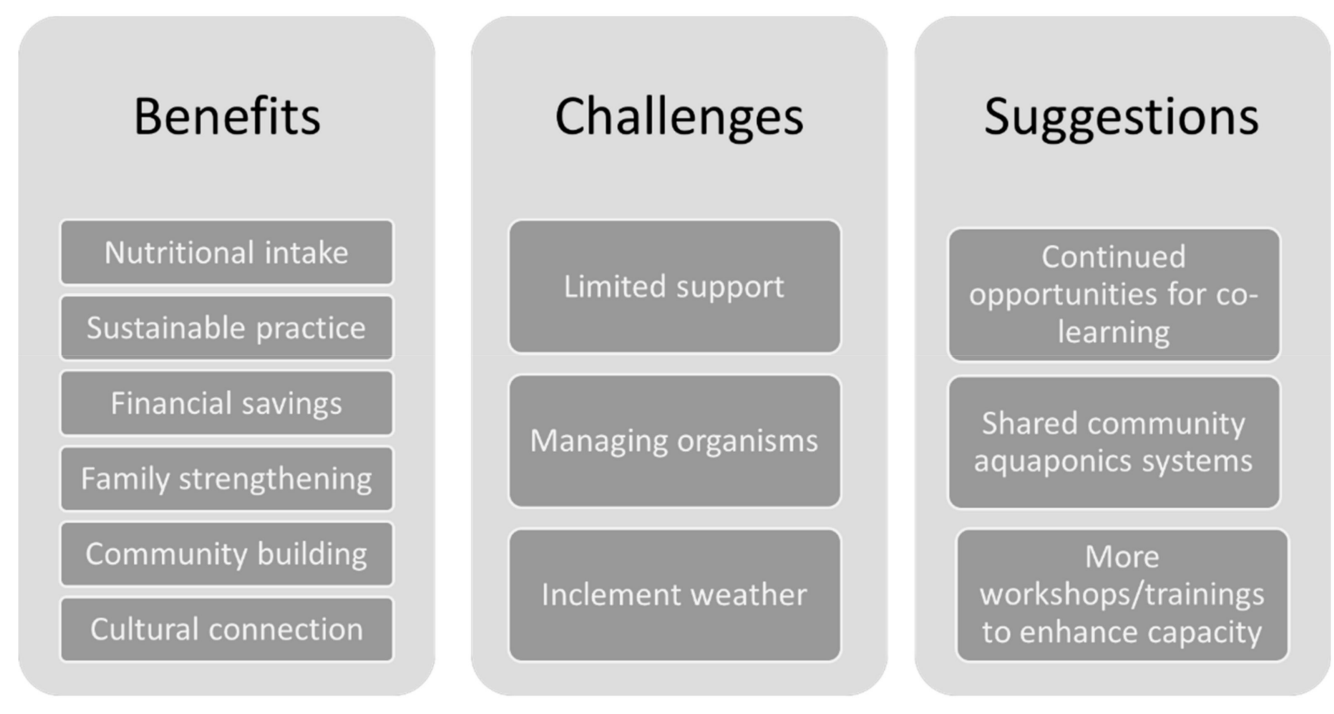

Figure 2. Themes from Waimānalo aquaponics interviews. 


\subsection{Benefits}

The majority of participants, including those with partially functioning and nonfunctioning systems, stated that aquaponics far exceeded their expectations and identified multiple benefits that they gained from their systems. These included health benefits, financial savings, family strengthening, community connectedness, environmental sustainability, and cultural 'aina-based practices. Participants commented that their diet improved because of the availability of fresh produce from their aquaponics systems. Many stated that their system served as a reminder to stay healthy by eating the plants and fish that were growing in their own backyards. One participant commented, "Everyone should have one. We grow all our herbs, eat fish once a week, and are able to feed the rabbit and chickens with the plants from the system." The participants also reported that their aquaponics systems helped them economically by not only saving money on their grocery bills but also by selling their extra produce. One participant indicated that she saved $\$ 60$ a month on the cost of buying lettuce because of their backyard system. Another participant shared that their family sells turmeric, which is valued for its medicinal properties, to neighbors and local markets.

Additionally, the participants reported that the aquaponics systems became a place for family strengthening, enhanced community connectedness, and a deepened understanding of Native Hawaiian cultural practices that are grounded in the importance of 'aina. Within the families, the participants stated that the aquaponics systems gave their children an opportunity to learn how to take care of the plants and fish, which helped instill a sense of responsibility and accomplishment. It also provided a way for family members to spend quality time with one another by working on a project with a shared goal. This in turn helped family members to prepare and cook the produce together and enjoy meals with one another. A participant with a non-functioning system mentioned that when his aquaponics system was working, the system helped him bond with his son. He stated, "My son's 7th grade science fair project was on aquaponics, which is what initially got us involved in the systems, which helped us become closer by having to work on a project together." Another participant who is interested in starting a system also mentioned the strengthening of a family bond. "Our family works on our backyard garden with our nephews and dad. Aquaponics would be another project for us to do together."

Participants reported that their systems exceeded many of their expectations as the plants grew quickly and often produced more than a single family could handle. Even participants who had non-functioning systems spoke positively about their previous system and its abilities to produce a large amount of food quickly. Many of the participants reported that the aquaponics systems allowed them to give back to their community by sharing their excess produce and fish with neighbors and even donating them to local food banks.

The systems also served as a way for participants to connect with Hawaiian culture by growing plants and herbs used in lā'au lapa'au, a traditional and spiritual form of healing that stems from the Hawaiian perspective that food and plants are medicine. One participant stated, "aquaponics allows me to grow traditional herbs which offer me food and medicine, it also gives me a space for mental growth and renewal." Additionally, the participants shared that the aquaponics system served as an example of sustainable agriculture and energy. All of the five current system users mentioned the low cost of system maintenance; for some participants the systems were solar powered, which further cut down on the electricity costs.

These benefits mirrored the expected outcomes of those who were interested in obtaining a system in the near future. They expressed that their interests in aquaponics were based on their desire to grow their own food sustainably, to share with their family and neighbors, strengthen family bonds, and learn about lā'au lapa'au.

\subsection{Challenges}

Some participants reported facing technical and logistical challenges in maintaining or setting up their system. They reported mechanical issues, primarily damage to pipes, trays, and tables. Water 
maintenance was also a challenge, as the water needed to be at a specific $\mathrm{pH}$ and temperature for the fish. One participant's $\mathrm{pH}$ kit was stolen from her yard, and others mentioned complications in the water temperature due to uncontrolled temperature and sunlight. Overproduction of fish was cited as a problem since many of the families did not frequently eat tilapia, which is the most common fish grown in aquaponics systems in Hawai'i. A participant stated that "the amount of fish was overwhelming, I was not sure what to do with them and gave them away because they reproduce so quickly." Figuring out the proper plants to grow also served as a challenge for some participants. One individual reported that her trays broke from planting a papaya tree. Others commented on the challenges of controlling invasive roots of tomatoes and spearmint. While aquaponics systems tend to be exposed to less weeds and pests, participants still mentioned a variety of bugs and diseases that they needed to be aware of and learn how to control. The participants also shared that unpredictable weather, such as strong winds, presented challenges to maintaining their aquaponics systems and in some cases destroyed their systems and all their produce. Sudden storms also caused electrical shortages, which caused fish tank pump to stop functioning and, in some cases, all of the fish to die.

\subsection{Suggestions}

Participants readily provided suggestions on how families can be supported in starting and sustaining their aquaponics systems. These suggestions centered on building an aquaponics community of learners. All participants were interested in learning from others' experiences, particularly about which plants grow best and how to maintain their aquaponics systems and how to prevent leaks in the pipes. In addition, they suggested using Facebook groups, group text, group email, and newsletters to communicate with one another as well as organize monthly community workshops for those engaged in aquaponics. When asked what type of workshops they are interested in, a participant indicated interest in learning about moon phases, fish reproduction cycles, water quality testing, and system management. Other topics mentioned included algae control, plant diseases, best plants to grow, cooking and lā'au lapa'au classes, using their aquaponics produce, and fish population control. Participants asked that these workshops be hands-on and interactive. Participants who are active with their aquaponics systems indicated that they would be willing to participate in future aquaponics workshops to help build new systems for others in their community.

\section{Discussion}

The findings from this study suggest that there are many benefits of having a backyard aquaponics system in Waimānalo. This could lead to further strengthening the interest in aquaponics as a health intervention within the Waimānalo community and potentially other rural and indigenous communities who share similar values of land, health, and community. Although there is no existing literature that examines aquaponics as a health intervention, there are many parallels between our findings with emerging gardening literature. Similar to our findings that having a backyard aquaponics system helped families make healthier choices related to their eating habits, engaging in gardening has been shown to increase the consumption of fruit and vegetables (Berti et al. 2003; Davis et al. 2011; Masset et al. 2012; McAleese and Rankin 2007). Another study found an increase in the availability of fruits and vegetables (146\%) and an increase in the consumption of fruits $(28 \%)$ and vegetables $(33 \%)$ among children and families participating in the Growing Healthy Kids (GHK) program (Castro et al. 2013). Additionally, participants in another study reported emotional and mental health benefits while taking care of their gardens and harvesting the produce (Hawkins et al. 2011).

Other benefits related to economic sufficiency and community connectedness identified by this study reflect findings from other similar gardening studies where participants increased their income by selling their produce to markets or restaurants and donated excess produce to senior citizens or homeless shelters (Draper and Freedman 2010). Additionally, a community garden project with aboriginal people in Canada found that their project also provided families emotional support both within and beyond the project (Mundel and Chapman 2010). The project emphasized participant 
health, including a connection to Mother Earth and the Universe. Similar to our study, the participants in the Canadian program felt a greater sense of land stewardship and gained a deeper understanding of the reciprocal relationship between humans and nature. As evidenced by the benefits and suggestions, the aquaponics project goes beyond solely increasing the intake of fruits and vegetables and increasing food accessibility in the Waimānalo community. It offers a way to engage and reconnect with Native Hawaiian cultural practices of ecological sustainability and land stewardship that are grounded in health and community.

Challenges identified by the participants were related to technical and logistical difficulties, such as managing the plants and fish in the systems and unpredictable weather. Although most studies on community gardening focus on the positive aspects of gardening, our participants readily shared a broad range of their experiences with the mindset that their ideas would enhance efforts related to aquaponics. This may have been due to the strong existing partnership and trust between the community partners and university researchers. As with any project, there is a learning curve with setting up a functional and sustainable system. Our participants willingly shared their struggles with their aquaponics experience and provided thoughtful suggestions.

Many suggestions centered on building an aquaponics community of learners where participants could exchange ideas with one another through in-person and interactive workshops. Community members would then be able to build their relationships with one another, engage in cooking and lā'au lapa'au demonstrations using their aquaponics produce, and learn about technical issues related to maintaining their aquaponics systems. Similarly, participants in the community garden project with aboriginal people in Canada suggested having a community kitchen and community gardening activities to promote organic fruits and vegetables as well as traditional foods and to organize cultural celebrations and feasts (Mundel and Chapman 2010). The participants' suggestions focused on ways to expand the project to include more members of the community, such as having a shared community aquaponics system that would enhance accessibility and attending workshops where they could share their experiences and learn from each other. The funding acquired by GCW from multiple local agencies was limited in amount $(\$ 2500-\$ 25,000)$ and had short grant periods (within one year) and did not allow for ongoing support of all the families at the time. However, the aquaponics effort in Waimānalo has recently evolved into a clinical research study using community-based participatory research principles and indigenous methodologies (Ho-Lastimosa et al. 2019). The goal of this research initiative is to systematically document the health and social outcomes of participating in these workshops as well as to provide ongoing support and resources for family participants. It is recommended that communities who are interested in initiating a similar aquaponics program consider the importance of providing ongoing support and resources for families.

\section{Limitations}

Limitations of this study include a small sample size due to the limited time for data collection and inability to interview more Waimānalo families with an aquaponics system. Social desirability bias is also a possibility although many suggestions and challenges were freely shared by the participants. There is also the possibility of interviewer bias, as different researchers facilitated the interviews and the completeness of an interview could vary. However, there were many strengths of this project, including the use of CBPR approaches. Interview questions were framed and approved by community partners and the idea of the project came from the community as they were interested in how aquaponics has benefited the families and the community and how they can continue to be supported. The qualitative method is another strength of the study as it emphasizes face-to-face interactions and the oral tradition of Native Hawaiian culture. It allowed participants to share and expand on their perspectives that were not limited by the researchers' knowledge.

Following CBPR principles, results from this study were orally shared with the participants at a community gathering. Participants not only validated the accuracy of the themes but also shared their appreciation for the opportunity to share their knowledge and perspectives. Future directions for 
this project include obtaining more funding to continue providing aquaponics systems to interested families as well as providing ongoing support to current families using the suggestions given by the participants of the study. Future plans are to continue examining how aquaponics can be a health intervention by continuing to conduct interviews with more families and to develop appropriate quantitative indicators to measure the impacts.

\section{Conclusions}

While specific to the community of Waimānalo, the findings of this study can act as a guideline for other community aquaponics projects. The overall consensus of having a backyard aquaponics system was overwhelmingly positive. Many participants, including those with partially-functioning and nonfunctioning systems, reported that having an aquaponics system far exceeded their expectations. This study demonstrates that aquaponics brought both financial and health benefits for individual families as well as for the wider Waimānalo community. It strengthened relationships throughout the community, within families, among neighbors, and with the aina. It also addressed the issue of food insecurity in Waimannalo by increasing access to, and the consumption of, fresh fruits and vegetables. In doing so, participants were able to create positive changes in their health and wellbeing.

In terms of future direction, there are opportunities for growth and improvement in aquaponics in Waimānalo. Additional funding has been obtained to further test the aquaponics workshop as a public health intervention with more families (Ho-Lastimosa et al. 2019). With more workshops and a clear path of communication, this community project can easily grow and be available to more families. It could expand to other communities and be the start of an aquaponics curriculum that is adopted to encourage healthy and ancient practices of food sustainability. Previous participants could serve as peer leaders for future workshops and aid others in the setup and maintenance of their systems. Backyard aquaponics systems have exciting potential to strengthen community wellness.

Author Contributions: Conceptualization and resources, J.K.B., I.H.-L., and J.J.C.-D.; methodology, validation, formal analysis, investigation, data curation, writing-original draft preparation, J.K.B., Y.A., G.M., A.L.C.B., I.N.K., N.C., and R.P.; writing-review and editing, J.K.B., Y.A., I.H.-L., G.M., D.E., M.S.S., and J.J.C.-D.; supervision and project administration, I.H.-L. and J.J.C.-D. All authors have read and agreed to the published version of the manuscript.

Funding: This research received no external funding.

Conflicts of Interest: The authors declare no conflict of interest.

\section{References}

Beamer, Kamanamaikalani. 2014. No Mākou Ka Mana: Liberating the Nation. Hawai'i: Kamehameha Publishing.

Berti, Peter R., Julia Krasevec, and Sian FitzGerald. 2003. A review of the effectiveness of agriculture intervention in improving nutrition outcomes. Public Health Nutrition 7: 599-609. [CrossRef] [PubMed]

Castro, Dian C., Margaret Samuels, and Ann Harman. 2013. Growing healthy kids. American Journal of Preventive Medicine 44: S193-99. [CrossRef] [PubMed]

Davis, Jaimie N., Emily E. Ventura, Lauren T. Cook, Lauren E. Gyllenhammer, and Nicole M. Gatto. 2011. LA Sprouts: A gardening, nutrition, and cooking intervention for Latino youth improves diet and reduces obesity. Journal of the American Dietetic Association 111: 1224-30. [CrossRef] [PubMed]

Department of Business Economic Development and Tourism. 2012. Increased Food Security and Food Self-Sufficiency Strategy. Available online: http://files.hawaii.gov/dbedt/op/spb/INCREASED_FOOD_ SECURITY_AND_FOOD_SELF_SUFFICIENCY_STRATEGY.pdf (accessed on 27 December 2019).

Draper, Carrie, and Darcy Freedman. 2010. Review and analysis of the benefits, purposes, and motivations associated with community gardening in the United States. Journal of Community Practice 18: 458-92. [CrossRef]

Goodyear-Ka'ōpua, Noelani, Ikaika Hussey, and Erin Kahunawaika'ala Wright. 2014. A Nation Rising: Hawaiian Movements for Life, Land, and Sovereignty. Durham: Duke University Press. 
Hawaii Department of Health. 2001. Hunger and food insecurity in Hawaii: Baseline Estimates Honolulu: Hawaii Department of Health. Available online: http://health.hawaii.gov/hhs/files/2013/04/specfood.pdf (accessed on 27 December 2019).

Hawkins, Jemma L, Katie J. Thirlaway, Karianne Backx, and Deborah A. Clayton. 2011. Allotment gardening and other leisure activities for stress reduction and healthy aging. Hort Technology 21: 577-85. [CrossRef]

Ho-Lastimosa, Ilima, Jane Chung-Do, Phoebe Hwang, Theodore Radovich, Ikaika Rogerson, Kenneth Ho, Samantha Keaulana, Joseph Keawe'aimoku Kaholokula, and Michael Spencer. 2019. Integrating Native Hawaiian tradition with the modern technology of aquaponics. Global Health Promotion 26: 87-92. [CrossRef] [PubMed]

Ho-Lastimosa, Ilima, Phoebe Hwang, and Bob Lastimosa. 2014. Community strengthening through canoe culture: Ho'omana'o Mau as method and metaphor. Hawaii Journal of Medicine \& Public Health 73: 397-99.

Israel, Barbara, Amy Schulz, Edith Parker, and Adam Becker. 1998. Review of Community-Based (Research: Assessing partnership approaches to improve public health. Annual Review of Public Health and doi:10 1146) Research: Assessing partnership approaches to improve public health. Annual Review of Public Health 19: 173-202. [CrossRef] [PubMed]

Johnson, David, Neil Oyama, Loic LeMarchand, and Lynne Wilkens. 2004. Native Hawaiians mortality, morbidity, and lifestyle: comparing data from 1982, 1990, and 2000. Pacific Health Dialogue 11: 120-30.

Kaholokula, Joseph Keawe'aimoku, Andrea Nacapoy, and Kā’ohimanu Dang. 2009. Social justice as a public health imperative for kanaka maoli. Alternative 5: 117-37. [CrossRef]

Kame'eleihiwa, Lilikalā. 1992. Native Land and Foreign Desires: Pehea LA E Pono Ai? How Shall We Live in Harmony? Honolulu: Bishop Museum Press.

Kame'eleihiwa, Lilikalā. 2016. Kaulana O'ahu Me He 'Āina Momona Mamuli O Nā Ha'awina 'Aumākua E Kahea Aku Nei, "Hui, e hele mai 'ai, a'ai i ka mea loa'a!". In Food and Power in Hawaii: Visions of Food Democracy. Edited by Aya Hirata Kimura and Krisnawati Suryanata. Hawai'i: University of Hawai'i Press.

Look, Mele, Mililani Trask-Batti, Robert Agres, Marjorie Mau, and Joseph Keawe'aimoku Kaholokula. 2014. Assessment and Priorities for Health $\mathcal{E}$ Wellness in Native Hawaiians $\mathcal{E}$ Other Pacific Peoples. Hawai'i: University of Hawai'i.

Masset, Edoardo, Lawrence Haddad, Alexander Cornelius, and Jairo Isaza-Castro. 2012. Effectiveness of agricultural interventions that aim to improve nutritional status of children: Systematic review. British Medicine Journal 344: d822. [CrossRef] [PubMed]

Mau, Marjorie, Kara Wong, Jimmy Efird, Margaret West, Erin Saito, and Jay Maddock. 2008. Environmental factors of obesity in communities with native Hawaiians. Hawaii Journal of Medicine and Public Health 67: 233-36.

McAleese, Jessica, and Linda Rankin. 2007. Garden-based nutrition education affects fruit and vegetable consumption in sirith-grade adolescents. Journal of the American Dietetic Association 107: 662-65. [CrossRef] [PubMed]

McGregor, Davianna Pōmaika'i, Paula Morelli, Jon Matsuoka, Rona Rodenhurst, Noella Kong, and Michael Spencer. 2003. An ecological model of Native Hawaiian well-being. Pacific Health Dialog 10: 106-28. [PubMed]

Möhlenkamp, Paula, Charles Kaiaka Beebe, Margaret McManus, Angela Hi'ilei Kawelo, Keli'iahonui Kotubetey, Mirielle Lopez-Guzman, Craig Nelson, and Rosanna 'Anolani Alegado. 2018. Kū Hou Kuapā: Cultural Restoration Improves Water Budget and Water Quality Dynamics in He'eia Fishpond. Sustainability 11: 161. [CrossRef]

Mundel, Erika, and Gwen Chapman. 2010. A decolonizing approach to health promotion in Canada: The case of the urban aboriginal community kitchen garden project. Health Promotion International 25: 166-73. [CrossRef] [PubMed]

Rezentes, William, III. 1996. Ka Lama Kukui Hawaiian Psychology: An Introduction. Honolulu: A'ali'i Books.

Tokunaga, Kanae, Clyde Tamaru, Harry Ako, and PingSun Leung. 2013. Economics of commercial aquaponics in Hawaii. Available online: http://www.ctsa.org/files/publications/HawaiiAquaponicsEconomics_Nov2013. pdf (accessed on 27 December 2019).

U.S. Census Bureau. 2015. State and County Quickfacts: Waimānalo, CDP Hawai'i. Available online: http://www.census.gov/quickfacts/table/PST045215/1578050,00 (accessed on 27 December 2019). 
Waimānalo Hawaiian Homestead Association. 2019. Available online: http://waimanalohha.org/ (accessed on 27 December 2019).

World Food Programme. 2016. What Is Food Security? Available online: https://www.wfp.org/node/359289 (accessed on 27 December 2019).

(1) (1) (C) 2020 by the authors. Licensee MDPI, Basel, Switzerland. This article is an open access article distributed under the terms and conditions of the Creative Commons Attribution (CC BY) license (http://creativecommons.org/licenses/by/4.0/). 